\title{
INNATE IMMUNITY
}

\section{Caspase-12: the long and the short of it}

New research published in Nature indicates that some people carry a single nucleotide polymorphism in the gene encoding caspase-12. This confers hyporesponsiveness to the bacterial cell-wall component lipopolysaccharide (LPS) and subsequently an enhanced susceptibility to bacterial invasion of the bloodstream (septicaemia) and increased mortality.

The polymorphism, which is confined to individuals of African descent, results in the production of a full-length caspase-12 precursor protein (Csp12-L), instead of a truncated caspase-12 protein (Csp12-S). Because other human caspases are involved in two processes - cytokine maturation (which occurs during inflammation through the cleavage of precursors) or apoptosis Saleh and colleagues examined the whole blood of donors of African origin for apoptotic and inflammatory responsiveness. The presence of Csp12-L correlated with a reduction in LPS-induced cytokine production, whereas both concanavalin-Astimulated production of most cytokines and apoptotic sensitivity were similar in individuals expressing either variant, indicating that caspase- 12 modulates the release of cytokines in response to LPS.

However, although caspase- 12 is related to the 'inflammatory' caspases involved in cytokine maturation, neither variant showed any proteolytic activity. The authors suggest that Csp12-L might function as a dominantnegative regulator of the activity of other caspases, potentially by antagonizing the inflammasome (the protein scaffold within which active cytokines are produced) and the associated pro-inflammatory pathways. This is supported by evidence that cell lines transfected with Csp12-L show decreased activation of the main pro-inflammatory transcription factor, nuclear factor- $\kappa B$ (NF-KB ). Csp12-S was also found to inhibit NF- $\kappa B$ activation, although to a lesser extent than Csp12-L.

The polymorphism in caspase- 12 is clearly functionally important because preliminary evidence shows that carrying the gene encoding Csp12-L is associated with

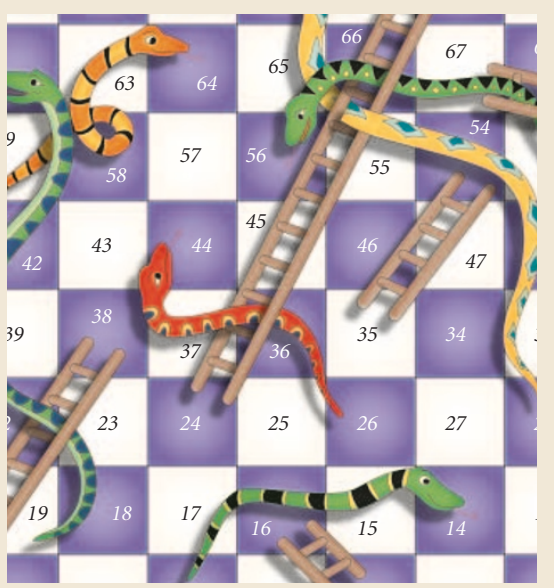

susceptibility to severe septicaemia and a subsequent increase in mortality. The authors suggest that caspase- 12 antagonists might have therapeutic potential for treating septic shock (which results from the overproduction of cytokines in response to LPS) and other inflammatory disorders.

Davina Dadley-Moore

(2) References and links

ORIGINAL RESEARCH PAPER Saleh, M. et al. Differential modulation of endotoxin responsiveness by human caspase-12 polymorphisms. Nature 429, 75-79 (2004) FURTHER READING Tracey, K. J. \& Warren, H. S. An inflammatory issue. Nature 429, 35-36 (2004)

\section{Langerhans cells in the frame}

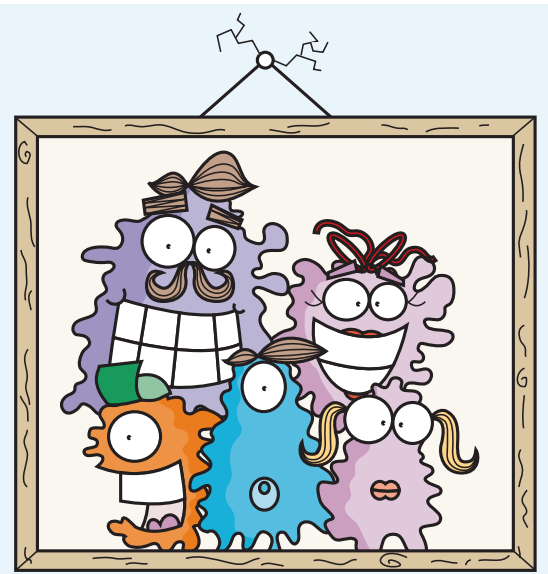

The occurrence of graft-versus-host disease (GVHD) in the skin of patients receiving bonemarrow transplants is a common and serious complication, hindering the widespread use of this procedure. Although donor T cells are known to have an active role in GVHD, it is unclear which cells are important in triggering the disease-causing (alloreactive) T-cell response. Now, a study in Nature Medicine by Miriam Merad and colleagues reveals that host Langerhans cells that persist in the skin following bone-marrow transplantation are responsible for the induction of GVHD.

To study the role of Langerhans cells - the main population of antigen-presenting cells in the skin - in GVHD, lethally irradiated mice were transplanted with allogeneic bone marrow and the fate of Langerhans cells assessed. When $\mathrm{T}$ cells were depleted from the bone-marrow transplant, host Langerhans cells persisted in the skin, even though the blood and spleen were repopulated with donor-derived dendritic cells. By contrast, when $T$ cells were included in the transplant, host Langerhans cells were replaced by donor-derived cells. The authors show that this occurred by two donor-T-cell-mediated processes: FAS ligand (CD95L)-mediated depletion of host Langerhans cells and the induction of expression of inflammatory chemokines in the skin, which recruit donor Langerhans-cell precursors.
However, mice that received whole bone marrow still developed GVHD, because the depletion of host Langerhans cells occurred after donor $\mathrm{T}$ cells cause disease. So, the authors developed a strategy to eliminate host Langerhans cells before donor T-cell infusion. First, irradiated mice were reconstituted with T-cell-depleted bone marrow, allowing complete chimerism to be established in the blood but not the skin. These mice were then exposed to ultraviolet light, which caused host Langerhans cells to migrate out of the skin and allowed repopulation with donor-derived cells. After several weeks, the mice were then retransplanted with bone marrow containing T cells. No GVHD developed in these mice, indicating that Langerhans-cell replacement in the skin is required to prevent GVHD following transplantation of T cells.

Future experiments will address whether exposing patients to ultraviolet light before transplantation might help prevent GVHD in human recipients.

Lucy Bird

(2) References and links

ORIGINAL RESEARCH PAPER Merad, M. et al. Depletion

of host Langerhans cells before transplantation of donor alloreactive T cells prevents skin graft-versus-host disease. Nature Med. 10, 510-517 (2004)

FURTHER READING Emerson, S. G. Tanning before transplant: lancing the Langerhans cell. Nature Med. 10 451-452 (2004) 